\title{
UMA NOVA PROPOSTA PARA O CONTROLE DE INTERCÂMBIO ENTRE ÁREAS
}

\author{
Marcelo José dos Santos \\ mjdsantos@yahoo.com.br
}

\author{
José Luiz Rezende Pereira \\ jluiz@ieee.org
}

\author{
João Alberto Passos Filho \\ jopass@cepel.br
}

Edimar José de Oliveira

edimardacee.ufje.br
Ivo Chaves da Silva Jr.

ivojeterra.com.br

UFJF / Faculdade de Engenharia

Juiz de Fora, MG

Brasil

\begin{abstract}
This paper describes a new methodology to evaluate the area interchange control (AIC) in a power flow problem using the Newton-Raphson method. In this methodology, the equations of the AIC are incorporated into the system of equations of the power flow problem, where the new state variables are the active power generation increments at the AIC regulating buses. Thus an augmented system of equations, which is linearized and solved at each iteration, is obtained. In addition, a new approach for the representation of multiple AIC regulating buses at a given area is also presented. The proposed method has been tested and compared with existing methods in the literature using both small and large scale power systems. The results presented validate the proposed technique.
\end{abstract}

KEYWORDS: Area interchange control, power flow problem, augmented system.

\section{RESUMO}

Este trabalho descreve uma nova metodologia para o controle de intercâmbio entre áreas (CIA) em um problema

Artigo Submetido em 24/02/03

1a. Revisão em 28/04/03;

2a. Revisão em 22/01/04;

Aceito sob recomendação do Editor Associado

Prof. Dr. Glauco Taranto de fluxo de potência utilizando o método de NewtonRaphson. Nesta metodologia, as equações do CIA são incorporadas ao sistema de equações do problema tradicional de fluxo de potência, onde as novas variáveis de estado são os incrementos de geração de potência ativa das barras de folga. Obtém-se assim, um sistema aumentado de equações que é linearizado e resolvido a cada iteração. Adicionalmente, uma proposta para a representação de múltiplas barras de folga em uma mesma área é também apresentada. A metodologia proposta foi testada e comparada com outros métodos existentes na literatura usando-se sistemas de pequeno e grande porte. Os resultados apresentados validam a metodologia proposta.

PALAVRAS-CHAVE: Controle de intercâmbio entre áreas, problema de fluxo de potência, sistema aumentado.

\section{NOMENCLATURA}

$n b:$ número de barras.

$V \angle \theta$ : coordenadas polares de tensão.

$P_{f}$ : potência ativa líquida injetada na barra $f$.

$P_{L f}$ : carga ativa da barra $f$.

$P I_{i}^{e s p}$ : intercâmbio líquido especificado da área $i$. 
$P I_{i}^{\text {calc }}$ : intercâmbio líquido da área $i$, calculado a cada iteração.

$P_{G f \lim }$ : limite de geração de potência ativa da barra $f$.

$P_{G f_{0}}$ : geração inicial de potência ativa da barra $f$.

$g_{k m}+j b_{k m}$ : admitância série da linha $k-m$.

$h:$ contador de iterações.

Os vetores estão sublinhados.

\section{INTRODUÇÃO}

O fluxo de potência é uma das ferramentas mais utilizadas tanto no planejamento quanto na operação de sistemas elétricos de potência. Vários trabalhos têm sido publicados nesta área (Tinney e Hart, 1967; Stott, 1974; Stott e Alsac, 1974; Fuerte-Esquivel e Acha, 1997; Gotham e Heydt, 1998; Da Costa et alii, 1998).

Com o desenvolvimento dos sistemas elétricos de potência, torna-se cada vez mais importante controlar o fluxo de potência nas linhas de transmissão, de acordo com as necessidades de transferência de potência. Por outro lado, o rápido desenvolvimento das tecnologias de eletrônica de potência como os FACTS (Flexible AC Transmission Systems), vêm, por sua vez, demandando o desenvolvimento de modelos para análise de fluxo de potência em regime permanente (Fuerte-Esquivel e Acha, 1997; Gotham e Heydt, 1998; Fang e Ngan, 1999; FuerteEsquivel et alii, 2000; Duan et alii, 2000; Li et alii, 2000).

Com a desverticalização e o aumento da competição do setor elétrico no Brasil e no mundo, as empresas de energia elétrica têm investido em novas tecnologias visando o aumento da qualidade de energia a um custo otimizado. Esta mudança tem demandado o desenvolvimento de novas ferramentas computacionais que permitam a realização do CIA e a máxima capacidade de transferência de potência entre sub-sistemas (Xiao e Song, 2000, Verma et alii, 2001, Feng e Shrestha, 2001).

O CIA é estabelecido através de contratos entre as concessionárias de energia elétrica. Utilizando o método das tensões nodais em coordenadas polares, existem na literatura basicamente dois métodos para a realização do CIA: o primeiro, conhecido como método de ajustes alternados (Stagg e El-Abiad, 1968), no qual após a resolução do sistema de equações e a atualização dos módulos e ângulos das tensões, obtêm-se os resíduos de intercâmbio líquido. Estes, por sua vez, são utilizados para corrigir as potências ativas geradas das barras de folga das áreas; o segundo, incorpora as equações do CIA, na forma linearizada, internamente à matriz Jacobiana (Monticelli, 1983), obtendo-se uma matriz Jacobiana com dimensões aumentadas. Neste método, as novas variáveis de estado são os incrementos de ângulo de tensão das barras de folga.

Neste artigo, o CIA é também representado internamente à matriz Jacobiana. No entanto, as novas variáveis de estado são os incrementos de potência ativa gerada das barras de folga de cada área. A grande vantagem desta metodologia quando comparada com a referência Monticelli (1983) é que a matriz Jacobiana torna-se mais esparsa, sendo também o modelo para representação de múltiplas barras de folga em uma mesma área mais simples. A metodologia proposta foi validada utilizando-se sistemas elétricos de grande porte.

\section{CONSIDERAÇÕES INICIAIS}

Nos sistemas elétricos de potência, onde as redes são interligadas, é necessário que sejam controlados os intercâmbios (fluxos de potência ativa nas linhas) entre todas as áreas que compõem o sistema. Em uma rede com $n$ áreas são controlados os intercâmbios de $n-1$ áreas, já que o intercâmbio de uma delas fica definido pelas demais (primeira lei de Kirchhoff), ou seja, é a área de referência. $\mathrm{A}$ barra do tipo $\mathrm{V} \theta$ está incluída na área de referência.

O intercâmbio líquido de uma área é definido como a soma algébrica dos fluxos de potência ativa nas linhas que interligam essa área com as demais. As exportações são admitidas positivas e as importações negativas.

Em cada área (exceto a área de referência) é necessário deixar pelo menos uma barra de geração com potência ativa livre. Estas barras são denominadas barras de folga e são classificadas como do tipo $\mathrm{V}$, onde somente os módulos das tensões nodais são especificados.

O objetivo do CIA é regular o intercâmbio total de cada área, ou seja, as injeções de potência ativa das barras de folga são ajustadas para manter os intercâmbios líquidos das áreas nos seus valores especificados.

É importante destacar que, caso uma ou mais áreas não possuam barras de folga, os seus intercâmbios líquidos não podem ser controlados durante o processo iterativo.

A figura 1 ilustra um sistema elétrico composto por $n$ áreas. 


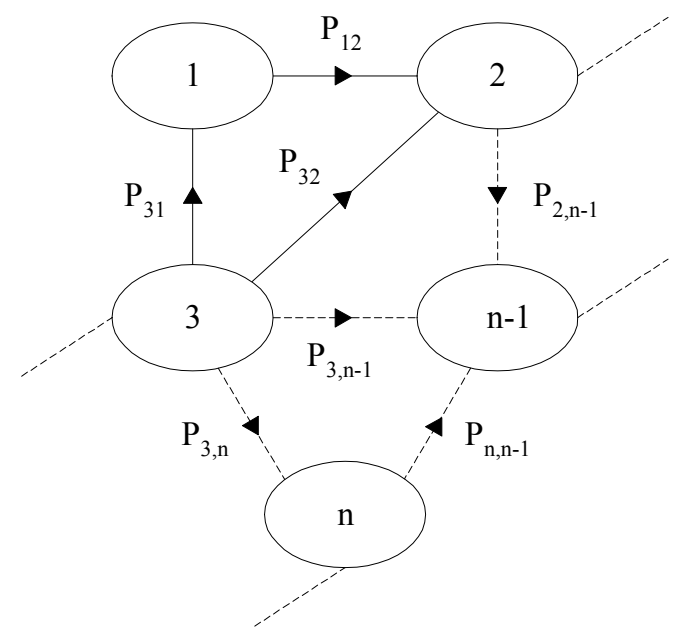

Figura 1 - Exemplo de um sistema com $n$ áreas

Considerando que a área $n$ seja a área de referência, tem-se que os intercâmbios líquidos das áreas restantes são:

$$
\begin{gathered}
P I_{1}=P_{12}-P_{31} \\
P I_{2}=-P_{12}-P_{32}+\ldots+P_{2, n-1} \\
P I_{3}=P_{31}+P_{32}+\ldots+P_{3, n-1}+P_{3, n} \\
\vdots \\
P I_{i}=\ldots \\
\vdots \\
P I_{n-1}=-P_{2, n-1}-P_{3, n-1}-P_{n, n-1}+\ldots
\end{gathered}
$$

\section{METODOLOGIA PROPOSTA}

$\mathrm{Na}$ metodologia proposta, o CIA é realizado através da incorporação de equações de controle de intercâmbio na forma linearizada, no sistema de equações do problema geral de fluxo de potência em coordenadas polares. Com isso, tem-se um novo sistema de equações aumentado. O número de equações do CIA é igual ao número de áreas que têm seus intercâmbios líquidos controlados durante o processo iterativo.

Nesta metodologia, as novas variáveis de estado são os incrementos de potência ativa gerada das barras de folga $\left(\Delta P_{G i}\right)$. Assim, as potências ativas geradas das barras de folga são atualizadas a cada iteração, de tal forma que o intercâmbio líquido de cada área seja atendido ao final do processo iterativo.

Seja $i$ uma área cujo intercâmbio líquido deve ser mantido no seu valor especificado.
O intercâmbio líquido da área $i$ é definido pela equação (5):

$$
P I_{i}^{e s p}=\sum_{\substack{m \in \Omega_{k} \\ m \notin i}} P_{k m}
$$

onde:

$k$ : barra de intercâmbio da área $i$.

$\Omega_{k}$ : conjunto de barras diretamente conectadas à barra $k$, porém não pertencente à área $i$.

A expressão para o fluxo de potência ativa (intercâmbio) na linha $k-m$ é dada pela equação

(6):

$$
P_{k m}=V_{k}^{2} g_{k m}-V_{k} V_{m}\left(g_{k m} \cos \theta_{k m}+b_{k m} \operatorname{sen} \theta_{k m}\right)
$$

Linearizando-se a equação (5), obtém-se:

$$
\Delta P I_{i}=\frac{\partial P I_{i}}{\partial \underline{\theta}} \cdot \Delta \underline{\theta}+\frac{\partial P I_{i}}{\partial \underline{V}} \cdot \Delta \underline{V}
$$

onde:

$$
\Delta P I_{i}=P I_{i}^{e s p}-P I_{i}^{c a l c}
$$

No método de Newton-Raphson são usados como critérios de convergência do sistema, os resíduos relativos às potências ativa e reativa. Com a inclusão do CIA, considera-se um critério adicional para sua convergência, ou seja, o valor de $\left|\Delta P I_{i}\right|$ de cada área deve ser menor do que uma tolerância pré-estabelecida.

A equação (9) representa a forma compacta do sistema aumentado a ser resolvido a cada iteração pelo método de Newton-Raphson.

$$
\left[\begin{array}{c}
\Delta \underline{P} \\
\Delta \underline{Q} \\
\Delta \underline{P I}
\end{array}\right]=\left[\begin{array}{cc:c}
H & N & \frac{\partial \underline{P}}{\partial P_{G}} \\
J & L & \frac{\partial \underline{Q}}{\partial P_{G}} \\
\hdashline \frac{\partial \underline{P I}}{\partial \underline{\theta}} & \frac{\partial \underline{P I}}{\partial \underline{V}} & \frac{\partial \underline{P I}}{\partial \underline{P_{G}}}
\end{array}\right] \cdot\left[\begin{array}{c}
\Delta \underline{\theta} \\
\Delta \underline{\underline{P_{G}}}
\end{array}\right]
$$

onde:

$\Delta \underline{P}, \Delta \underline{Q}, H, N, J, L, \Delta \underline{\theta}$ e $\Delta \underline{V}$ são encontrados em Monticelli (1983).

$\triangle \underline{P I}: \quad$ vetor dos resíduos relativos aos intercâmbios líquidos conforme é definido na equação (8). 
$\frac{\partial \underline{P I}}{\partial \underline{\theta}}$ e $\frac{\partial \underline{P I}}{\partial \underline{V}}:$

vetores das derivadas dos intercâmbios líquidos em relação aos ângulos e módulos das tensões, respectivamente. As derivadas não nulas somente aparecem nas posições correspondentes às barras envolvidas nos intercâmbios, ou seja, se a linha $k-m$ é de intercâmbio, as derivadas não serão nulas nas posições $k$ e $m$.

$\Delta \underline{P_{G}}: \quad$ vetor das variáveis de estado referentes ao CIA, que são neste modelo, representadas pelos incrementos de geração de potência ativa das barras de folga.

$\frac{\partial \underline{P}}{\partial \underline{P_{G}}}$ : $\quad$ vetor das derivadas de potência ativa injetada em relação às variáveis de estado referentes ao CIA. Neste caso, as derivadas não são nulas apenas nas posições correspondentes às barras de folga.

$\frac{\partial \underline{Q}}{\partial P_{G}}$ : $\quad$ vetor das derivadas de potência reativa injetada em relação às variáveis de estado referentes ao CIA. Neste caso, as derivadas são sempre nulas. $\begin{array}{ll}\frac{\partial \underline{P I}}{\partial P_{G}}: & \begin{array}{l}\text { vetores das derivadas dos intercâmbios } \\ \text { líquidos em relação às variáveis de }\end{array}\end{array}$ estado referentes ao CIA. Neste caso, são sempre nulas, pois os intercâmbios são funções apenas dos fluxos de potência ativa nas linhas.

Seja $f$ a barra de folga da área $i$. A forma linearizada da equação de potência ativa da barra $f$ é dada pela equação (10):

$$
\Delta P_{f}^{\prime}=P_{G f}^{(h)}-P_{L f}-P_{f}^{(h)}=-\Delta P_{G f}+\frac{\partial P_{f}}{\partial \underline{\theta}} \cdot \Delta \underline{\theta}+\frac{\partial P_{f}}{\partial \underline{V}} \cdot \Delta \underline{V}
$$

A cada iteração, a potência ativa gerada da barra de folga $f$ é atualizada como mostra a equação (11):

$$
P_{G f}^{(h+1)}=P_{G f}^{(h)}+\Delta P_{G f}^{(h)}
$$

É interessante notar que os elementos das colunas adicionais da matriz Jacobiana são todos nulos, exceto nas posições correspondentes às injeções de potência ativa das barras de folga, onde as derivadas são iguais a -1 , conforme mostrado na equação (10). Como consequência, a matriz Jacobiana aumentada apresenta poucos elementos não nulos em comparação à metodologia apresentada em Monticelli (1983), onde aparecem derivadas não nulas nas posições correspondentes às barras do sistema que estão conectadas às barras de folga.

\subsection{Exemplo llustrativo}

Considere a figura 2 que representa um sistema de 4 barras e 3 áreas. As barras 4 e 2 são as barras de folga das áreas 1 e 2 , respectivamente, e a área 3 é a área de referência. Os sentidos dos intercâmbios estão representados pelas setas.

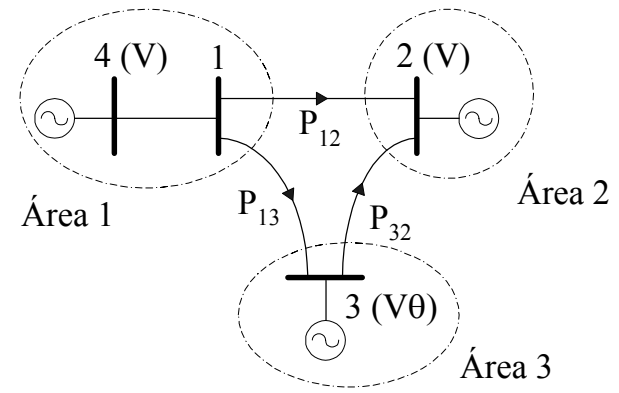

Figura 2 - Exemplo de um sistema de 4 barras e 3 áreas

Os intercâmbios líquidos das áreas 1 e 2 são, respectivamente:

$$
\begin{gathered}
P I_{1}=P_{12}+P_{13} \\
P I_{2}=-P_{12}-P_{32}
\end{gathered}
$$

Os resíduos de intercâmbio líquido das áreas 1 e 2 são dados, respectivamente, por:

$$
\begin{aligned}
& \Delta P I_{1}=P I_{1}^{e s p}-P I_{1} \\
& \Delta P I_{2}=P I_{2}^{e s p}-P I_{2}
\end{aligned}
$$

A equação (16) representa o sistema aumentado a ser resolvido a cada iteração pelo método de Newton-Raphson.

$$
\left[\begin{array}{c}
\Delta P_{1} \\
\Delta Q_{1} \\
\Delta P_{2}^{\prime} \\
\Delta P_{4}^{\prime} \\
\Delta I_{1} \\
\Delta I_{2}
\end{array}\right]=\left[\begin{array}{cccc:cc}
H_{11} & N_{11} & H_{12} & H_{14} & 0 & 0 \\
J_{11} & L_{11} & J_{12} & J_{14} & 0 & 0 \\
H_{21} & N_{21} & H_{22} & 0 & -1 & 0 \\
H_{41} & N_{41} & 0 & H_{44} & 0 & -1 \\
\hdashline C_{11} & D_{11} & C_{12} & 0 & 0 & 0 \\
C_{21} & D_{21} & C_{22} & 0 & 0 & 0
\end{array}\right] \cdot\left[\begin{array}{c}
\Delta \theta_{1} \\
\Delta V_{1} \\
\Delta \theta_{2} \\
\Delta \theta_{4} \\
\Delta P_{G 2} \\
\Delta P_{G 4}
\end{array}\right]
$$

Os elementos referentes às linhas adicionais, correspondentes às equações de intercâmbio na forma linearizada, são dados por:

$$
\begin{aligned}
& C_{11}=\frac{\partial P I_{1}}{\partial \theta_{1}}=\frac{\partial\left(P_{12}+P_{13}\right)}{\partial \theta_{1}} \\
& D_{11}=\frac{\partial P I_{1}}{\partial V_{1}}=\frac{\partial\left(P_{12}+P_{13}\right)}{\partial V_{1}}
\end{aligned}
$$




$$
\begin{gathered}
C_{12}=\frac{\partial P I_{1}}{\partial \theta_{2}}=\frac{\partial\left(P_{12}+P_{13}\right)}{\partial \theta_{2}} \\
C_{21}=\frac{\partial P I_{2}}{\partial \theta_{1}}=\frac{\partial\left(-P_{12}-P_{32}\right)}{\partial \theta_{1}} \\
D_{21}=\frac{\partial P I_{2}}{\partial V_{1}}=\frac{\partial\left(-P_{12}-P_{32}\right)}{\partial V_{1}} \\
C_{22}=\frac{\partial P I_{2}}{\partial \theta_{2}}=\frac{\partial\left(-P_{12}-P_{32}\right)}{\partial \theta_{2}}
\end{gathered}
$$

Uma característica desta metodologia é que as equações de potência ativa das barras de folga são mantidas nesta formulação, o que mantém a matriz Jacobiana original inalterada.

$\mathrm{Na}$ equação (16), somente as equações de potência reativa das barras de folga 2 e 4 devem ser eliminadas. Já para a barra de referência 3 , além da equação de potência reativa também deve-se eliminar a equação de potência ativa.

$\mathrm{Na}$ equação (16), dos elementos das colunas adicionais, apenas nas linhas correspondentes às equações de potência ativa das barras de folga 2 e 4 , aparecem elementos não nulos. O número total de elementos não nulos é igual ao número total de barras de folga do sistema. Já com relação às linhas adicionais, apenas os elementos das colunas correspondentes às barras que fazem parte dos intercâmbios não são nulos, ou seja, nas posições das barras 1 e 2 .

Ao final de cada iteração do processo de solução, as potências ativas geradas das barras de folga 2 e 4 são atualizadas da seguinte maneira:

$$
\begin{aligned}
& P_{G 2}^{(h+1)}=P_{G 2}^{(h)}+\Delta P_{G 2}^{(h)} \\
& P_{G 4}^{(h+1)}=P_{G 4}^{(h)}+\Delta P_{G 4}^{(h)}
\end{aligned}
$$

\section{MÚLTIPLAS BARRAS DE FOLGA NUMA MESMA ÁREA}

A metodologia proposta permite a consideração de mais de uma barra de folga por área para a realização do CIA, de uma maneira bem flexível, pois as novas variáveis de estado continuam sendo os incrementos de potência ativa gerada das barras de folga. Esta modelagem é similar ao caso de apenas uma barra de folga por área, a não ser pela inclusão de equações de controle adicionais.

A contribuição de geração de potência ativa de cada barra de folga é dada pelo seu fator de participação, em relação à subsequente barra de folga.
Seja $i$ uma área composta por $n f$ barras de folga. Logo, temse as seguintes relações de geração de potência ativa:

$$
\begin{gathered}
P_{G 1}=\alpha_{12} \cdot P_{G 2} \\
P_{G 2}=\alpha_{23} \cdot P_{G 3} \\
\vdots \\
P_{G(n f-1)}=\alpha_{(n f-1) n f} \cdot P_{G n f}
\end{gathered}
$$

As relações entre os fatores de participação das $n f$ barras de folga são obtidas através das seguintes expressões:

$$
\begin{gathered}
\alpha_{12}=\frac{\alpha_{1}}{\alpha_{2}}=\frac{P_{G 1_{0}}}{P_{G 2_{0}}} \\
\alpha_{23}=\frac{\alpha_{2}}{\alpha_{3}}=\frac{P_{G 2_{0}}}{P_{G 3_{0}}} \\
\vdots \\
\alpha_{(n f-1) n f}=\frac{\alpha_{(n f-1)}}{\alpha_{n f}}=\frac{P_{G(n f-1)_{0}}}{P_{G n f_{0}}}
\end{gathered}
$$

Os fatores de participação podem ser definidos pelo usuário, e são geralmente associados a custos de geração que são obtidos de dados de planejamento do sistema.

Reescrevendo a equação (25) na forma linearizada, obtêmse as novas equações de controle a serem incorporadas ao sistema de equações:

$$
\begin{gathered}
\Delta g_{1}=P_{G 1}^{(h)}-\alpha_{12} \cdot P_{G 2}^{(h)}=-\Delta P_{G 1}+\alpha_{12} \cdot \Delta P_{G 2} \\
\Delta g_{2}=P_{G 2}^{(h)}-\alpha_{23} \cdot P_{G 3}^{(h)}=-\Delta P_{G 2}+\alpha_{23} \cdot \Delta P_{G 3} \\
\vdots \\
\Delta g_{(n f-1)}=P_{G(n f-1)}^{(h)}-\alpha_{(n f-1) n f} \cdot P_{G n f}^{(h)}= \\
-\Delta P_{G(n f-1)}+\alpha_{(n f-1) n f} \cdot \Delta P_{G n f}
\end{gathered}
$$

As atualizações das potências ativas geradas das barras de folga são obtidas pela equação (11).

É interessante salientar que a inclusão de múltiplas barras de folga no modelo de CIA, que é a principal contribuição deste trabalho, permite verificar a capacidade máxima de transferência de potência de uma área para outra usando um programa de fluxo de potência e fluxo de potência continuado, onde as barras de folga podem ser consideradas as barras onde há incremento de geração para suprimento da carga no processo de continuação. 


\subsection{Exemplo Ilustrativo}

Considere a figura 3 que representa um sistema de 5 barras e 3 áreas. A barra 4 é a barra de folga da área 1 e as barras 2 e 5 são as barras de folga da área 2 . A área 3 é a referência.

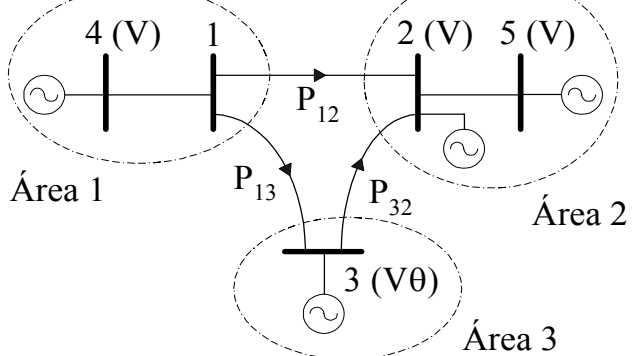

Figura 3 - Exemplo de um sistema de 5 barras e 3 áreas

Os intercâmbios líquidos e os respectivos resíduos das áreas 1 e 2 são dados pelas equações (12), (13), (14) e (15).

A nova equação de controle descrita na forma linearizada é dada por:

$$
\Delta g_{1}=P_{G 2}^{(h)}-\alpha_{25} \cdot P_{G 5}^{(h)}=-\Delta P_{G 2}+\alpha_{25} \cdot \Delta P_{G 5}
$$

O resíduo correspondente à equação (28) é dado por:

$$
\Delta g_{1}=P_{G 2}^{(h)}-\alpha_{25} \cdot P_{G 5}^{(h)}
$$

A equação (30) representa o sistema aumentado a ser resolvido a cada iteração pelo método de Newton-Raphson.

$$
\left[\begin{array}{c}
\Delta P_{1} \\
\Delta Q_{1} \\
\Delta P_{2}^{\prime} \\
\Delta P_{4}^{\prime} \\
\Delta P_{5}^{\prime} \\
\Delta P I_{1} \\
\Delta P I_{2} \\
\Delta g_{1}
\end{array}\right]=\left[\begin{array}{ccccc:ccc}
H_{11} & N_{11} & H_{12} & H_{14} & 0 & 0 & 0 & 0 \\
J_{11} & L_{11} & J_{12} & J_{14} & 0 & 0 & 0 & 0 \\
H_{21} & N_{21} & H_{22} & 0 & H_{25} & -1 & 0 & 0 \\
H_{41} & N_{41} & 0 & H_{44} & 0 & 0 & -1 & 0 \\
0 & 0 & H_{52} & 0 & H_{55} & 0 & 0 & -1 \\
\hdashline C_{11} & D_{11} & C_{12} & 0 & 0 & 0 & 0 & 0 \\
C_{21} & D_{21} & C_{22} & 0 & 0 & 0 & 0 & 0 \\
0 & 0 & 0 & 0 & 0 & -1 & 0 & \alpha_{25}
\end{array}\right] \cdot\left[\begin{array}{c}
\Delta \theta_{1} \\
\Delta V_{1} \\
\Delta \theta_{2} \\
\Delta \theta_{4} \\
\Delta \theta_{5} \\
\Delta P_{G 2} \\
\Delta P_{G 4} \\
\Delta P_{G 5}
\end{array}\right]
$$

Os elementos referentes às linhas adicionais, correspondentes às equações de intercâmbio na forma linearizada, são dados pelas equações (17) a (22).

\section{CONSIDERAÇÃO DOS LIMITES DE GERAÇÃO DE POTÊNCIA ATIVA DAS BARRAS DE FOLGA}

Quando as injeções de potência ativa das barras de folga violam seus limites, elas têm seus valores de geração de potência ativa especificados nos respectivos limites violados, deixam de ser barras de folga e passam a ser do tipo PV.
Se uma área qualquer do sistema possui apenas uma barra de folga e se a mesma violar seu limite de geração de potência ativa, o intercâmbio líquido desta área deixa de ser mantido no seu valor especificado. Isto é feito, retirando-se do problema a equação de intercâmbio correspondente à esta área. Por outro lado, se uma área possui mais de uma barra de folga e apenas uma delas não violar seu limite de geração de potência ativa, o intercâmbio líquido desta área é mantido através da injeção de potência ativa nesta barra de folga. Considere novamente o sistema mostrado na figura 3 . Se somente a barra de folga 2 violar o seu limite de geração de potência ativa, o sistema aumentado a ser resolvido a cada iteração é dado pela equação (31):

$$
\left[\begin{array}{c}
\Delta P_{1} \\
\Delta Q_{1} \\
\Delta P_{2}^{\prime} \\
\Delta P_{4}^{\prime} \\
\Delta P_{5}^{\prime} \\
\Delta P I_{1} \\
\Delta P I_{2}
\end{array}\right]=\left[\begin{array}{ccccc:cc}
H_{11} & N_{11} & H_{12} & H_{14} & 0 & 0 & 0 \\
J_{11} & L_{11} & J_{12} & J_{14} & 0 & 0 & 0 \\
H_{21} & N_{21} & H_{22} & 0 & H_{25} & 0 & 0 \\
H_{41} & N_{41} & 0 & H_{44} & 0 & -1 & 0 \\
0 & 0 & H_{52} & 0 & H_{55} & 0 & -1 \\
\hdashline C_{11} & D_{11} & C_{12} & 0 & 0 & 0 & 0 \\
C_{21} & D_{21} & C_{22} & 0 & 0 & 0 & 0
\end{array}\right] \cdot\left[\begin{array}{c}
\Delta \theta_{1} \\
\Delta V_{1} \\
\Delta \theta_{2} \\
\Delta \theta_{4} \\
\Delta \theta_{5} \\
\Delta P_{G 4} \\
\Delta P_{G 5}
\end{array}\right]
$$

A geração de potência ativa da barra 2 é dada por:

$$
P_{G 2}=P_{G 2 \lim }
$$

No entanto, se ambas as barras de folga 2 e 5 violarem seus limites de geração de potência ativa, o itercâmbio líquido da área 2 deixa de ser controlado. O sistema aumentado é, então, dado pela equação (33):

$$
\left[\begin{array}{c}
\Delta P_{1} \\
\Delta Q_{1} \\
\Delta P_{2}^{\prime} \\
\Delta P_{4}^{\prime} \\
\Delta P_{5}^{\prime} \\
\Delta P_{1}^{\prime}
\end{array}\right]=\left[\begin{array}{ccccc:c}
H_{11} & N_{11} & H_{12} & H_{14} & 0 & 0 \\
J_{11} & L_{11} & J_{12} & J_{14} & 0 & 0 \\
H_{21} & N_{21} & H_{22} & 0 & H_{25} & 0 \\
H_{41} & N_{41} & 0 & H_{44} & 0 & -1 \\
0 & 0 & H_{52} & 0 & H_{55} & 0 \\
\hdashline C_{11} & D_{11} & C_{12} & 0 & 0 & 0
\end{array}\right] \cdot\left[\begin{array}{c}
\Delta \theta_{1} \\
\Delta V_{1} \\
\Delta \theta_{2} \\
\Delta \theta_{4} \\
\Delta \theta_{5} \\
\Delta P_{G 4}
\end{array}\right]
$$

A geração de potência ativa da barra 2 é dada pela equação (32) e a da barra 5 é dada por:

$$
P_{G 5}=P_{G 5 \lim }
$$

\section{ASPECTOS COMPUTACIONAIS}

As linhas/colunas adicionais da matriz Jacobiana são representadas como barras fictícias do sistema elétrico e os elementos não nulos destas linhas/colunas são representados por linhas de transmissão fictícias. Esta estratégia adotada permite o uso de técnicas de esparsidade usando o algoritmo de ordenação proposto por Tinney II (Tinney e Walker, 1967) com certa facilidade para o sistema aumentado. $\mathrm{O}$ modelo proposto foi implementado no programa ANAREDE de propriedade do CEPEL. 


\section{RESULTADOS}

Para a validação do método proposto foram utilizados três sistemas testes: (i) um sistema fictício de pequeno porte, contendo 11 barras, 14 linhas e 3 áreas; (ii) um sistema equivalente da região Sudeste do sistema elétrico brasileiro, contendo 730 barras, 1146 linhas e 12 áreas e (iii) um sistema equivalente das regiões Sul-Sudeste do sistema elétrico brasileiro, contendo 1768 barras, 2523 linhas e 23 áreas.

Foram admitidos que os resíduos das equações de fluxo de potência devem ser menores que 0,0001 p.u. e que os resíduos referentes à todas as equações de controle devem ser menores que 0,0001 p.u.. Além disto, foram consideradas duas situações distintas: (i) uma barra de folga por área e (ii) múltiplas barras de folga por área: considerando ou não, os limites de geração de potência ativa das mesmas.

\subsection{Uma barra de folga por área}

\section{Sistema teste de 11 barras}

A figura 4 representada no apêndice A ilustra este sistema, sendo a área 2 a referência. Os sentidos dos intercâmbios estão representados pelas setas. O problema converge em 3 iterações utilizando o método proposto, e os intercâmbios nas linhas do sistema valem: $P_{63}=-22.0 \mathrm{MW}$, $P_{11,3}=14.2 \mathrm{MW}, P_{35}=16.2 \mathrm{MW}$ e $P_{11,5}=9.8 \mathrm{MW}$.

Os intercâmbios líquidos das áreas 1 e 3 são, respectivamente:

$$
\begin{gathered}
P I_{1}=-P_{63}+P_{35}-P_{11,3}=24.0 \mathrm{MW} \\
P I_{3}=P_{11,3}+P_{11,5}=24.0 \mathrm{MW}
\end{gathered}
$$

Já o intercâmbio líquido da área 2, pode ser obtido tanto pela primeira lei de Kirchhoff quanto pelo somatório dos intercâmbios que conectam essa área com as demais:

$$
P I_{2}=-P_{11,5}-P_{35}+P_{63}=-P I_{1}-P I_{3}=-48.0 \mathrm{MW}
$$

A tabela 1 mostra alguns resultados por área.

Tabela 1 - Barras de folga, intercâmbios líquidos, importações e exportações de cada área do sistema de 11 barras

\begin{tabular}{|c|c|c|c|c|}
\hline Área & $\begin{array}{c}\text { Barra } \\
\text { de } \\
\text { Folga }\end{array}$ & $\begin{array}{c}\text { Intercâmbio } \\
\text { Líquido } \\
\text { (MW) }\end{array}$ & $\begin{array}{c}\text { Importações } \\
\text { (MW) }\end{array}$ & $\begin{array}{c}\text { Exportações } \\
\text { (MW) }\end{array}$ \\
\hline 1 & 4 & 24.0 & 14.2 & 38.2 \\
\hline 2 & -- & -48.0 & 48.0 & 0.0 \\
\hline 3 & 10 & 24.0 & 0.0 & 24.0 \\
\hline
\end{tabular}

Quando o problema é resolvido levando-se em conta a ordenação dos elementos pelo método de Tinney II, deve-se colocar um valor muito pequeno no lugar dos elementos nulos nas diagonais da matriz Jacobiana referentes à expansão. Isto, pode evitar problemas numéricos durante a fatoração. Nos testes realizados verificou-se que $1.0 \times 10^{-6}$ apresenta bons resultados.

\section{Sistemas testes de 730 e 1768 barras}

A tabela 2 mostra uma comparação entre os dois métodos existentes na literatura e o método proposto.

É importante destacar também que as mesmas rotinas de solução do sistema de equações foram utilizadas nas metodologias existentes na literatura e na proposta.

Tabela 2 - Comparação entre o método de ajustes alternados, o método apresentado em Monticelli (1983) e o método proposto

\begin{tabular}{|c|c|c|c|}
\hline \multirow{2}{*}{$\begin{array}{c}\text { Sistema } \\
\text { Teste }\end{array}$} & $\begin{array}{c}\text { Método de } \\
\text { Ajustes } \\
\text { Alternados }\end{array}$ & $\begin{array}{c}\text { Método apresentado } \\
\text { em Monticelli } \\
\text { (1983) }\end{array}$ & $\begin{array}{c}\text { Método } \\
\text { Proposto }\end{array}$ \\
\hline 730 & 6 & 4 & 4 \\
\hline 1768 & 7 & 4 & 4 \\
\hline
\end{tabular}

\subsection{Múltiplas barras de folga por área}

\section{Sistema teste de 11 barras}

Este sistema é idêntico ao da figura 4, exceto pela consideração das barras 1 e 7 como barras de folga adicionais das áreas 1 e 3, respectivamente. A tabela 3 mostra alguns resultados por área, após o sistema convergir em 3 iterações utilizando o método proposto.

A tabela 4 mostra alguns resultados por área, após o sistema convergir em 3 iterações utilizando o método proposto, e considerando que as barras de folga 1 e 7 tiveram seus limites de geração de potência ativa violados. Os valores dos limites violados estão representados em negrito.

Tabela 3 - Barras de folga, fator de participação, potência ativa gerada e intercâmbios líquidos do sistema de 11 barras

\begin{tabular}{|c|c|c|c|c|}
\hline \multirow{2}{*}{ Área } & $\begin{array}{c}\text { Barra } \\
\text { de } \\
\text { Folga }\end{array}$ & $\begin{array}{c}\text { Fator de } \\
\text { Participação } \\
\text { (\%) }\end{array}$ & $\begin{array}{c}\text { Pot. Ativa } \\
\text { Gerada } \\
\text { (MW) }\end{array}$ & $\begin{array}{c}\text { Intercâmbio } \\
\text { Líquido } \\
\text { (MW) }\end{array}$ \\
\hline \multirow{2}{*}{1} & 1 & 30.0 & 19.2 & \multirow{2}{*}{24.0} \\
\cline { 2 - 5 } & 4 & 70.0 & 44.9 & \\
\hline 2 & -- & -- & -- & -48.0 \\
\hline \multirow{2}{*}{3} & 7 & 60.0 & 31.0 & \multirow{2}{*}{24.0} \\
\cline { 2 - 4 } & 10 & 40.0 & 20.7 & \multicolumn{2}{|c}{} \\
\hline
\end{tabular}


Tabela 4 - Barras de folga, fator de participação, potência ativa gerada e intercâmbios líquidos do sistema de 11 barras, considerando os limites de geração de potência ativa das barras 1 e 7

\begin{tabular}{|c|c|c|c|c|}
\hline \multirow{2}{*}{ Área } & $\begin{array}{c}\text { Barra } \\
\text { de } \\
\text { Folga }\end{array}$ & $\begin{array}{c}\text { Fator de } \\
\text { Participação } \\
\text { (\%) }\end{array}$ & $\begin{array}{c}\text { Pot. Ativa } \\
\text { Gerada } \\
\text { (MW) }\end{array}$ & $\begin{array}{c}\text { Intercâmbio } \\
\text { Líquido } \\
\text { (MW) }\end{array}$ \\
\hline \multirow{2}{*}{1} & 1 & 28.1 & $\mathbf{1 8 . 0}$ & \multirow{2}{*}{24.0} \\
\cline { 2 - 4 } & 4 & 71.9 & 46.1 & \\
\hline 2 & -- & -- & -- & -48.0 \\
\hline \multirow{2}{*}{3} & 7 & 54.3 & $\mathbf{2 8 . 0}$ & \multirow{2}{*}{24.0} \\
\cline { 2 - 4 } & 10 & 45.7 & 23.6 & \\
\hline
\end{tabular}

De acordo com a tabela 4, pode-se observar que os intercâmbios líquidos foram mantidos, pois ainda existe uma barra de folga em cada área. No entanto, os fatores de participação são diferentes, dado que os geradores 1 e 7 encontram-se nos seus valores limites.

A tabela 5 mostra alguns resultados por área, após o sistema convergir em 4 iterações utilizando o método proposto, e considerando que as barras de folga 1 e 4 tiveram seus limites de geração de potência ativa violados. Os valores dos limites violados estão representados em negrito.

Tabela 5 - Barras de folga, fator de participação, potência ativa gerada e intercâmbios líquidos do sistema de 11 barras, considerando os limites de geração de potência ativa das barras 1 e 4

\begin{tabular}{|c|c|c|c|c|}
\hline \multirow{2}{*}{ Área } & $\begin{array}{c}\text { Barra } \\
\text { de } \\
\text { Folga }\end{array}$ & $\begin{array}{c}\text { Fator de } \\
\text { Participação } \\
\text { (\%) }\end{array}$ & $\begin{array}{c}\text { Pot. Ativa } \\
\text { Gerada } \\
\text { (MW) }\end{array}$ & $\begin{array}{c}\text { Intercâmbio } \\
\text { Líquido } \\
\text { (MW) }\end{array}$ \\
\hline \multirow{2}{*}{1} & 1 & 29.5 & $\mathbf{1 8 . 0}$ & \multirow{2}{*}{21.1} \\
\cline { 2 - 4 } & 4 & 70.5 & $\mathbf{4 3 . 0}$ & \\
\hline 2 & -- & -- & -- & -45.1 \\
\hline \multirow{2}{*}{3} & 7 & 60.0 & 31.0 & \multirow{2}{*}{24.0} \\
\cline { 2 - 4 } & 10 & 40.0 & 20.7 & \\
\hline
\end{tabular}

É interessante notar na tabela 5 que, o intercâmbio líquido da área 1 deixa de ser controlado, pois todas as barras de folga desta área tiveram seus limites de geração de potência ativa violados. Portanto, o valor determinado para o intercâmbio líquido da área 1 difere do seu respectivo valor especificado, conforme está representado nas tabelas 1, 3 e 4.

\section{Sistemas testes de 730 e 1768 barras}

A tabela 6 mostra uma comparação entre o método de ajustes alternados e o método proposto, quando são considerados os limites de geração de potência ativa em algumas barras de folga dos sistemas de 730 e 1768 barras.

A tabela 7 mostra os tempos computacionais correspondentes. Tendo em vista as dificuldades de implementação da referência Monticelli (1983) para múltiplas barras de folga, não foi possível fazer a comparação de resultados. Para a realização dos testes foi utilizado um microcomputador Pentium III de $730 \mathrm{Mhz}$ e $128 \mathrm{Mb}$ de memória.

\section{Tabela 6 - Comparação entre o método de ajustes alternados e o método proposto}

\begin{tabular}{|c|c|c|c|}
\hline \multirow{2}{*}{$\begin{array}{c}\text { Sistema } \\
\text { Teste }\end{array}$} & \multirow{2}{*}{$\begin{array}{l}\text { Número de Barras de } \\
\text { Folga com Limites de } \\
\text { Geração de Potência } \\
\text { Ativa Violados }\end{array}$} & \multicolumn{2}{|c|}{ Número de iterações } \\
\hline & & $\begin{array}{c}\text { Método de } \\
\text { Ajustes } \\
\text { Alternados }\end{array}$ & $\begin{array}{l}\text { Método } \\
\text { Proposto }\end{array}$ \\
\hline 730 & 6 & 18 & 4 \\
\hline 1768 & 8 & 24 & 4 \\
\hline
\end{tabular}

Tabela 7 - Tempos computacionais

\begin{tabular}{|c|c|c|}
\hline \multirow{2}{*}{$\begin{array}{c}\text { Sistema } \\
\text { Teste }\end{array}$} & \multicolumn{2}{|c|}{ Tempo computacional (s) } \\
\cline { 2 - 3 } & $\begin{array}{c}\text { Método de } \text { Ajustes } \\
\text { Alternados }\end{array}$ & Método Proposto \\
\hline 730 & 0.23 & 0.16 \\
\hline 1768 & 0.75 & 0.46 \\
\hline
\end{tabular}

\section{CONCLUSÕES}

O presente trabalho apresentou uma nova metodologia para a realização do CIA, o qual é introduzido internamente à matriz Jacobiana. Em comparação ao método de ajustes alternados, o método proposto é bem mais robusto computacionalmente. Já em comparação ao método mostrado em Monticelli (1983), apresenta diversas vantagens:

- A utilização do incremento $\Delta P_{G i}$ como variável de estado está mais próximo do sentido físico da atuação do sistema de controle, ou seja, o CIA é feito ajustando-se diretamente a potência ativa gerada dos geradores das barras de folga;

- A metodologia proposta permite o tratamento de múltiplas barras de folga por área, mantendo-se como variáveis de estado os incrementos de potência ativa gerada das barras de folga. Por outro lado, na metodologia apresentada em Monticelli (1983), a inclusão de múltiplas barras de folga torna a modelagem mais complexa, pelo fato de não se ter uma relação direta dos incrementos de potência ativa gerada das barras de folga no sistema de equações. Uma alternativa para implementação seria escrever as potências ativas geradas em função dos módulos e ângulos das tensões;

- O tratamento de múltiplas barras de folga por área se torna importante na manutenção do CIA, quando são considerados os limites de geração de potência ativa das mesmas, além de permitir que dados de 
planejamento de sistemas possam ser usados na definição dos fatores de participação de cada barra de folga em uma mesma área;

- A matriz Jacobiana original mantém-se inalterada e a matriz Jacobiana aumentada apresenta poucos elementos adicionais não nulos, ou seja, é uma matriz com alto grau de esparsidade.

\section{REFERÊNCIAS BIBLIOGRÁFICAS}

Da Costa, V. M., J. L. R. Pereira and N. Martins (1998). Developments in the Newton Raphson Power Flow Formulation Based on Current Injections. IEEE Transactions on Power Systems (November), Vol.15, No.4, pp.1320-1326.

Duan, X., J. Chen, F. Peng, Y. Luo and Y. Huang (2000). Power Flow Control with FACTS Devices. IEEE Power Engineering Society Summer Meeting (July), Seattle, USA, Vol.3, pp.1585-1589.

Fang, W. L. and H. W. Ngan (1999). Control Setting of Unified Power Flow Controllers Through a Robust Load Flow Calculation. IEE ProceedingsGeneration, Transmission and Distribution (July), Vol.146, No.4, pp.437-444.

Feng, W. and G. B. Shrestha (2001). Allocation of TCSC Devices to Optimize Total Transmission Capacity in a Competitive Power Market. IEEE Power Engineering Society Winter Meeting, Columbus, USA, Vol.2, pp.587-593.

Fuerte-Esquivel, C. R. and E. Acha (1997). A Newton-type Algorithm for the Control of Power Flow in Electrical Power Networks. IEEE Transactions on Power Systems (November), Vol.12, No.4, pp.14741480.

Fuerte-Esquivel, C. R., E. Acha and H. Ambriz-Pérez (2000). A Comprehensive Newton-Raphson UPFC Model for the Quadratic Power Flow Solution of Practical Power Networks. IEEE Transactions on Power Systems (February), Vol.15, No.1, pp.102109.

Gotham, D. J. and G. T. Heydt (1998). Power flow control and power flow studies for systems with FACTS devices. IEEE Transactions on Power Systems (February), Vol.13, No.1, pp.60-65.

Li, N., Y. Xu and H. Chen (2000). FACTS-Based Power Flow Control in Interconnected Power Systems. IEEE Transactions on Power Systems (February), Vol.15, No.1, pp.257-262.
Monticelli, A. (1983). Fluxo de Carga em Redes de Energia Elétrica. Edgar Blucher, São Paulo - SP.

Stagg, G. W. and A. H. El-Abiad (1968). Computer Methods in Power System Analysis. International Student Edition. McGraw-Hill.

Stott, B. and O. Alsac (1974). Fast Decoupled Load Flow. IEEE Transactions on Power Systems, PAS - 93, No.3, pp.859-869.

Stott, B. (1974). Review of Load Flow Calculation Methods. Proceedings of the IEEE, pp.916 - 929.

Tinney, W. F. and J. W. Walker (1967). Direct Solutions of Sparse Network Equations by Optimally Ordered Triangular Factorizations. Proceedings of the IEEE (November), No.55, pp.1801-1809.

Tinney, W. F. and C. E. Hart (1967). Power Flow Solution by Newton's Method. IEEE Transactions on Power Systems (November), PAS - 86, pp.1449 - 1456.

Verma, K. S., S. N. Singh and H. O. Gupta (2001). FACTS Devices Location for Enhancement of Total Transfer Capability. IEEE Power Engineering Society Winter Meeting, Columbus, USA, Vol.2, pp.522-527.

Xiao, Y. and Y. H. Song (2000). Available Transfer Capability (ATC) Evaluation by Stochastic Programming. IEEE Power Engineering Review (September), Vol.20, No.9, pp.50-52.

\section{APÊNDICE A - SISTEMA TESTE DE 11 BARRAS}

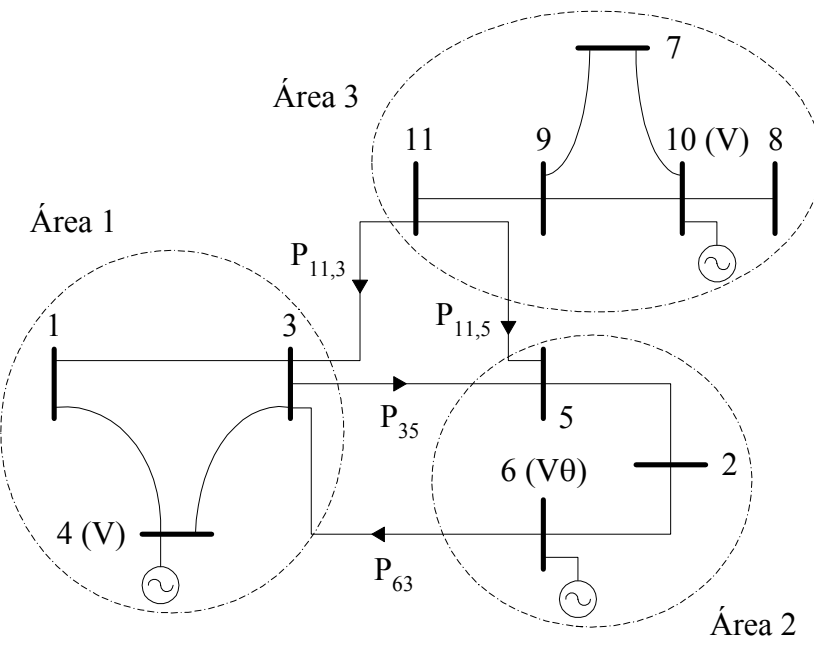

Figura 4 - Sistema de 11 barras 
Tabela 8 - Dados de barra

\begin{tabular}{|c|c|c|c|c|c|c|c|}
\hline No & Tipo & $\begin{array}{c}\text { Tensão } \\
\text { (p.u.) }\end{array}$ & $\begin{array}{c}\text { Ang. } \\
\text { (graus) }\end{array}$ & $\begin{array}{c}\text { Pger } \\
\text { (MW) }\end{array}$ & $\begin{array}{c}\text { Qger } \\
\text { (MVar) }\end{array}$ & $\begin{array}{c}\text { Pcar } \\
\text { (MW) }\end{array}$ & $\begin{array}{c}\text { Qcar } \\
\text { (MVar) }\end{array}$ \\
\hline 1 & PQ & 1.00 & 5.0 & 0.0 & 0.0 & 3.0 & 1.0 \\
\hline 2 & PQ & 0.90 & 4.0 & 0.0 & 0.0 & 15.0 & -8.0 \\
\hline 3 & PQ & 1.00 & 5.0 & 0.0 & 0.0 & 25.0 & 1.0 \\
\hline 4 & V & 1.00 & 0.0 & 0.0 & 0.0 & 10.0 & 0.0 \\
\hline 5 & PQ & 0.95 & 3.0 & 0.0 & 0.0 & 10.0 & -9.0 \\
\hline 6 & V & 0.95 & 0.0 & 0.0 & 0.0 & 25.0 & 0.0 \\
\hline 7 & PQ & 1.00 & 5.0 & 0.0 & 0.0 & 3.0 & 1.0 \\
\hline 8 & PQ & 0.90 & 4.0 & 0.0 & 0.0 & 15.0 & 5.0 \\
\hline 9 & PQ & 1.00 & 5.0 & 0.0 & 0.0 & 3.0 & 1.0 \\
\hline 10 & V & 1.00 & 0.0 & 0.0 & 0.0 & 2.0 & 0.0 \\
\hline 11 & PQ & 0.95 & 3.0 & 0.0 & 0.0 & 3.0 & 1.0 \\
\hline
\end{tabular}

Tabela 9 - Dados de linha

\begin{tabular}{|c|c|c|c|c|}
\hline De & Para & $\begin{array}{c}\text { Resistência } \\
\text { (p.u.) }\end{array}$ & $\begin{array}{c}\text { Reatância } \\
\text { (p.u.) }\end{array}$ & $\begin{array}{c}\text { Shunt } \\
\text { (p.u.) }\end{array}$ \\
\hline 6 & 2 & 0.99 & 0.10 & 0.0 \\
\hline 6 & 3 & 0.50 & 0.10 & 0.0 \\
\hline 3 & 5 & 0.50 & 0.10 & 0.0 \\
\hline 3 & 4 & 0.50 & 0.10 & 0.0 \\
\hline 1 & 3 & 0.50 & 0.10 & 0.0 \\
\hline 1 & 4 & 0.50 & 0.10 & 0.0 \\
\hline 2 & 5 & 0.50 & 0.10 & 0.0 \\
\hline 11 & 5 & 0.99 & 0.10 & 0.0 \\
\hline 11 & 3 & 0.50 & 0.10 & 0.0 \\
\hline 9 & 11 & 0.50 & 0.10 & 0.0 \\
\hline 9 & 10 & 0.50 & 0.10 & 0.0 \\
\hline 7 & 9 & 0.50 & 0.10 & 0.0 \\
\hline 7 & 10 & 0.50 & 0.10 & 0.0 \\
\hline 8 & 10 & 0.50 & 0.10 & 0.0 \\
\hline
\end{tabular}

